\title{
High Bandwidth Perovskite based Antenna for High- Resolution Biomedical Imaging at Terahertz
}

\author{
Abdoalbaset Abohmra ${ }^{1}$, \\ ${ }^{1}$ School of Electrical and Electronic Engineering, Glasgow \\ University, Glasgow, G12 8QQ, UK \\ Email: 2356006a@student.gla.ac.uk, \\ Syeda Fizzah Jilani ${ }^{2}$ \\ ${ }^{2}$ School of Electronic Engineering and Computer Science, \\ Queen Mary University of London, London E1 4NS, UK \\ Email:s.f.jillani@qmul.ac.uk,
}

\begin{abstract}
This paper suggests the prospective utilization of a hybrid perovskite, with a chemical name of Methylammonium Lead Iodide $\left(\mathrm{CH}_{3} \mathrm{NH}_{3} \mathrm{PbI}_{3}\right)$, in the design of a Terahertz (THz) antenna. A newly developed perovskite material has been used for the antenna design which can replace the conventional metallic patches. The proposed antenna operates in two THz bands, i.e. 3.6-7.4 THz and 8.25-10.0 THz. The realized gain of the antenna is above $4 \mathrm{dBi}$ and efficiency is above $70 \%$ in overall operating bandwidth.In addition, the radiation efficiency of the designed antenna could be improved by increasing the conductivity of perovskite film with the application of an external voltage. The reported performance of the antenna design provides the proof of the concept for the possible utilization of non-metallic advanced materials such as perovskite in manufacturing antennas for $\mathrm{THz}$ based biomedical applications.
\end{abstract}

Keywords-Bandwidth, Perovskite; Terahertz; wireless

\section{INTRODUCTION}

With the increasing demands of high-speed, reliable and secure wireless communication and relatively lesser influence of the radiations on the user, the research trend has emphasized on the migration towards high-frequency $\mathrm{THz}$ spectrum $[1,2]$. Recent research efforts have been focused to overcome the propagation and fabrication issues at $\mathrm{THz}$ and in developing THz sources, antennas, systems and applications [3]. In the past few years, the bandwidth of $0.1-10 \mathrm{THz}$ has gained attention for the analysis and is highly anticipated in the applications of bio-sensing and imaging, fast and secure wireless systems, and radar communication $[4,5]$. Numerous remarkable features of the $\mathrm{THz}$ band, for instance, high resolution of imaging, high confidentiality and data security, high penetrability of smoke or dust and wide available bandwidth, are promising for $\mathrm{THz}$ detectors, imaging and communication devices. Moreover, due to high absorption levels of $\mathrm{THz}$ waves by the water molecules and limited penetration ability into the human body, it has low tendency to produce hazardous ionization of biological tissues, and a favourable choice in the biomedical scanners.

A high-performance $\mathrm{THz}$ antenna is regarded as an essential unit, and several aspects need to be considered to develop an antenna design able to fulfil the demands of $\mathrm{THz}$ applications [6]. The progress has been made in the exploitation of novel materials instead of a copper-based radiating patch due to high

\author{
Hasan $\mathrm{Abbas}^{3}$ \\ ${ }^{3}$ Department of Physics, Texas A \& M University at Qatar, \\ Doha, Qatar \\ Email:hasan.abbas@qatar.tamu.edu \\ Akram Alomainy², \\ Email: a.alomainy@qmul.ac.uk \\ Muhammad Ali Imran ${ }^{1}$ and Qammer H. Abbasi ${ }^{1}$ \\ Email:
}

\{Muhammad.Imran,Qammer.Abbasi\}@glasgow.ac.uk

loss and degradation in efficiency as a result of a decrease in skin depth and conductivity at THz. Carbon-based materials i.e. graphene, and carbon nanotubes have found promising in this regard [7]. Perovskite compounds, due to the features such as superconductivity, ferroelectricity and low cost, have gained focus in various novel applications [8]. In this paper, the prospective perovskite antenna in $\mathrm{THz}$ bands is investigated.

\section{PEROVSKITE ANTENNA DESIGN}

The proposed antenna is designed and numerically analysed by using CST Microwave Studio (2018). The designed perovskite-based antenna is tested in simulation at the room temperature of $293 \mathrm{~K}$. Perovskite material is constructed in the CST by using the measured complex permittivity values demonstrated in [9]. Fig. 1 shows the antenna design with the dimensions $40 \times 40 \times 10 \mu \mathrm{m}^{3}$, consisting of a Perovskite based rectangular patch, while the ground plane and feedline are made of copper. Thin flexible film of polyamide is used as a substrate (dielectric constant, $\varepsilon_{r}=4.5$ and loss tangent, $\tan \delta=0.0027$ ).

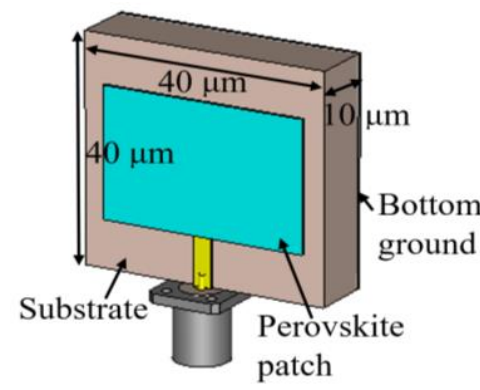

(a) (b)

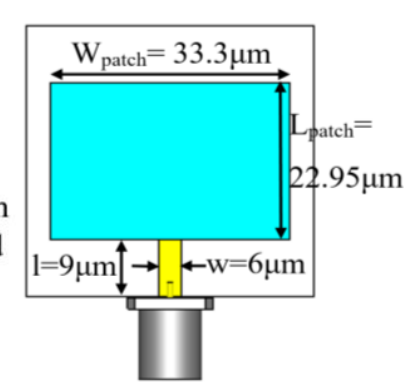

Fig. 1. Proposed perovskite-based THz antenna: (a) simulated antenna design; (b) Perovskite patch and copper feed line in the antenna prototype.

\section{RESULT AND DISCUSSION}

The reflection coefficient $\left(S_{11}\right)$ plot of Fig. 2 demonstrates two significant bands while taking $-10 \mathrm{~dB}$ as a reference. The band-I ranges from 3.6-7.4 THz and constitutes two dominant resonances at $5.18 \mathrm{THz}$ and $6.98 \mathrm{THz}$ respectively. The BandII covers the range of $8.25-10.0 \mathrm{THz}$ with an obvious resonant dip at $8.8 \mathrm{THz}$. The parametric analysis is carried out on the radiating length of the antenna and shown in Fig. 3. It has been 
observed that with the increase in length from $20.95 \mu \mathrm{m}$ to 29.7 $\mu \mathrm{m}$, the resonant frequency can be tuned towards lower range.

The gain and efficiency versus frequency plots are shown in Fig. 4, which indicates that the realized gain varies from 4-8 dBi in the overall operating range. The gain magnitude is above 4 $\mathrm{dBi}$ in the almost complete bandwidth. Simulated results of Fig. 4 also present good efficiency of $\sim 88 \%$ at $5.185 \mathrm{THz}$, which is comparable to the reported graphene-based THz antenna in [10].

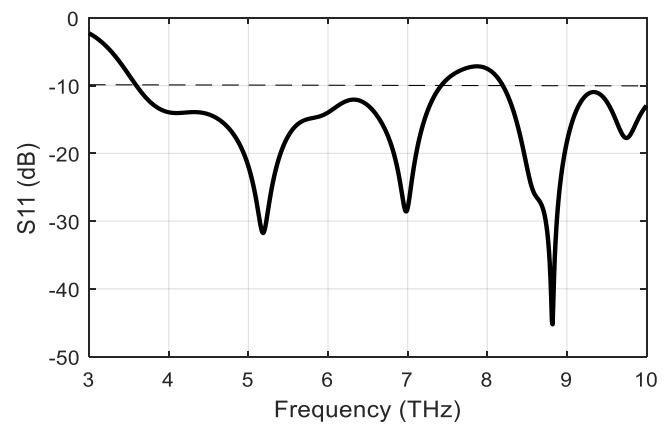

Fig. 2. Simulated $\mathrm{S}_{11}$ profile of the designed perovskite-based $\mathrm{THz}$ antenna.

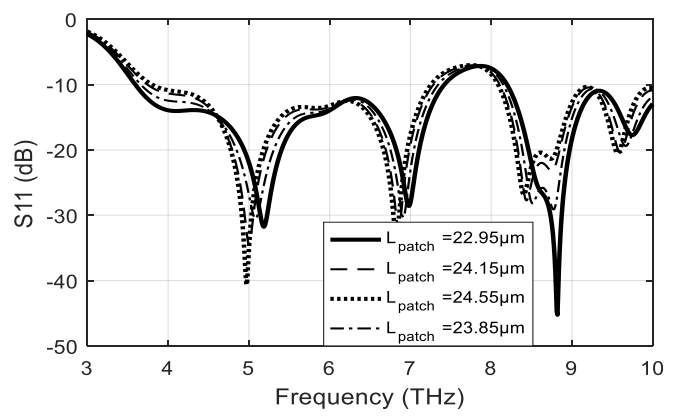

Fig. 3. Parametric analysis of the radiating length of the designed perovskitebased THz antenna.

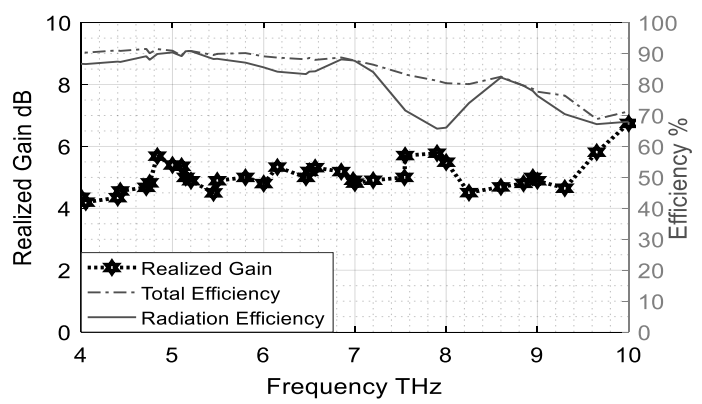

Fig. 4. Realized gain and efficiency plots of the proposed perovskite-based THz antenna.

The $\mathrm{E}$ and $\mathrm{H}$-plane radiation patterns of the designed antenna at the three major resonant frequencies are illustrated in Fig. 5. The main lobe with $4.53 \mathrm{dBi}$ is obtained at the resonance of $5.185 \mathrm{THz}$, while an increase in the magnitude of main lobe gain is observed at $6.985 \mathrm{THz}$ and $8.821 \mathrm{THz}$ resonances. Though the radiation is not perfect broadside in the complete operating range, yet this effect is obvious when the antenna is designed to cover a wide bandwidth. The high bandwidth, reasonable gain magnitudes and high efficiency depict that the perovskite can be deployed as a radiating element in the $\mathrm{THz}$ patch antennas.

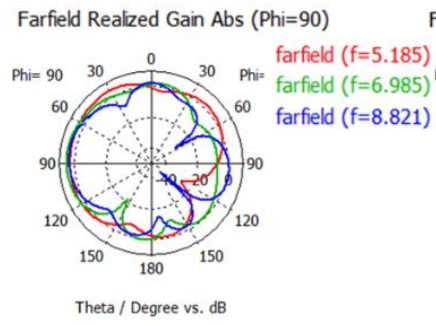

(a)
Farfield Realized Gain Abs (Phi=0)

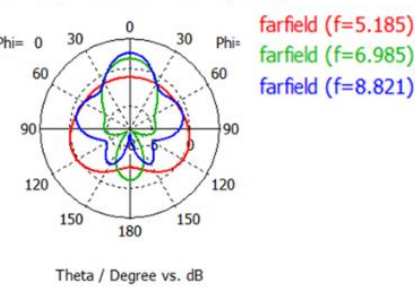

(b)
Fig. 5. Proposed perovskite-based THz antenna: (a) Bottom metallic ground of the simulated antenna; (b) Front view of the simulated antenna.

\section{CONCLUSION}

The $\mathrm{THz}$ antennas are anticipated to ensure a high-resolution imagining for the biomedical applications due to their short wavelength and high directivity. Novel materials have been developed for the potential deployment in modern $\mathrm{THz}$ systems to achieve high performance. The results show that the designed antenna covers two $\mathrm{THz}$ bands, i.e. $3.6-7.4 \mathrm{THz}$ and $8.25-10.0$ THz. The realized gain of the antenna is above $4 \mathrm{dBi}$ and radiation efficiency is above $70 \%$ in overall operating bandwidth. The antenna is regarded as a potential candidate for the future short-range $\mathrm{THz}$ communications.

\section{ACKNOWLEDGEMENT}

Sincere gratitude of the authors to the Libyan Government for this research support.

\section{REFERENCES}

[1] Ghann W, Uddin J. Terahertz (THz) Spectroscopy: A Cutting - Edge Technology. InTerahertz Spectroscopy-A Cutting Edge Technology 2017.

[2] M. S. Rabbani and H. Ghafouri-Shiraz, "Liquid crystalline polymer substrate-based THz microstrip antenna arrays for medical applications," IEEE Antennas Wireless Propag. Lett., vol. 16, pp. 1533-1536, 2017.

[3] P. Siegel, "Terahertz technology," IEEE Trans. Microw. Theory Techn., vol. 50, no. 3, pp. 910-928, 2002.

[4] L. Ranzani et al., "G-Band Micro-Fabricated Frequency-Steered Arrays With 2GHz Beam Steering," IEEE Trans. Terahertz Science Technology, vol. 3, no. 5, pp. 566-573, 2013.

[5] M. Khatib and M. Perenzoni, "Response optimization of antenna-coupled FET detectors for 0.85-to-1-THz imaging," IEEE Microw. Wireless Compon. Lett., vol. 28, no. 10, pp. 903-905, 2018.

[6] Bolin Chen and Huang Fengyi, "Research on a MEMS-technology-based corrugated horn antenna designed in Tera-Hertz regime," IEEE Int. Conf. Microw. Millimeter Wave Technol. (ICMMT), 2016, pp. 213-216.

[7] S. A. Naghdehforushha and G. Moradi, "Design of plasmonic rectangular ribbon antenna based on graphene for terahertz band communication," IET Microw., Antennas Propag., vol. 12, no. 5, pp. 804-807, 2018.

[8] C. L. Huang and Y. W. Tseng, "A low-loss dielectric using $\mathrm{CaTiO}_{3-}$ modified $\mathrm{Mg}_{1.8} \mathrm{Ti}_{1.1} \mathrm{O}_{4}$ ceramics for applications in dielectric resonator antenna," IEEE Trans. Dielectr. Electr. Insul., vol. 21, no. 5, pp. 22932300, 2014.

[9] M. A. Green, A. Ho-Baillie, and H. J. Snaith, "The emergence of perovskite solar cells," Nat. Photonics, vol. 8, no. 7, pp. 506, 2014.

[10] M. Dashti, and J. D. Carey, J.D., "Graphene microstrip patch ultrawide band antennas for THz communications," Adv. Funct. Mater., vol. 28, no. 11, pp.1705925, 2018. 